\title{
Advanced laboratory for testing plasma thrusters and Hall thruster measurement campaign
} Agnieszka Szelecka

\begin{abstract}
Plasma engines are used for space propulsion as an alternative to chemical thrusters. Due to the high exhaust velocity of the propellant, they are more efficient for long-distance interplanetary space missions than their conventional counterparts. An advanced laboratory of plasma space propulsion (PlaNS) at the Institute of Plasma Physics and Laser Microfusion (IPPLM) specializes in designing and testing various electric propulsion devices. Inside of a special vacuum chamber with three performance pumps, an environment similar to the one that prevails in space is created. An innovative Micro Pulsed Plasma Thruster $(\mathrm{L} \mu \mathrm{PPT})$ with liquid propellant was built at the laboratory. Now it is used to test the second prototype of Hall effect thruster (HET) operating on krypton propellant. Meantime, an improved prototype of krypton Hall thruster is constructed.
\end{abstract}

Key words: electric propulsion $\bullet$ Hall thruster

\author{
A. Szelecka \\ Institute of Plasma Physics and Laser Microfusion, \\ 23 Hery Str., 01-947 Warsaw, Poland, \\ Tel.: +48 22638 1005, Fax: +48 226668372 , \\ E-mail: agnieszka.szelecka@ifpilm.pl
}

Received: 12 October 2015

Accepted: 18 November 2015

\section{Introduction}

In all types of HETs, the quasi-neutral plasma is accelerated in the crossed magnetic and electric field. Due to the radial magnetic field, the electrons are magnetized, so their mobility along the axial electric field is considerably reduced. On the other hand, the magnetic field is too weak to influence the ions. In spite of the simple idea underlying the construction of such a thruster, the description of plasma discharge in this device is a complex problem. Therefore, the optimization in order to maximize its performance is a very difficult task [1]. In our case, the HETMAN code designed in our laboratory was used to study the operational characteristics of the thruster and, in particular, the dependence of the efficiency of the thruster on its parameters. This code relies on a 1D time-dependent hydrodynamical description of the discharge in the channel of the thruster. In order to run the code, many important parameters and material functions such as heat conductivity, anomalous electron transport, secondary electron emission, or complete geometry of the thruster and the magnetic field topology have to be provided.

In this article, we present the study of the determination of the best operating conditions for 
the second prototype of Krypton Large IMpulse Thruster (KLIMT), which is a $\sim 500 \mathrm{~W}$ class HET. We also discuss most important issues that appeared during the measurement campaign and their troubleshooting.

\section{Main problems with krypton thruster}

Krypton was chosen as a propellant because of its very lower price and greater abundance than the most popular xenon. On the other hand, the physical properties of krypton are quite close to that of xenon. The two main problems, which are encountered during the tests in the vacuum chamber, are overheating of some parts of the Hall thruster and sputtering from the chamber walls caused by the high-velocity krypton ions produced by the thruster.

\section{Overheating}

During the numerous tests, we observed the overheating of the gas supply line. The first attempt to avoid the anode line tube overheating was to assure a good thermal isolation between the anode connector and the gas feeding line. In KLIMT, the anode is also a gas distributor, so the gas connector is welded into the anode and becomes very hot during the thruster operation. For this reason, the special mechanical support, which serves simultaneously as a heat sink and a radiator, was designed.

Another problem associated with overheating was the design of the main radiator of the thruster. It turned out that in the first version of this radiator, some part of the radiated heat was emitted back to the thruster. To fix this design flaw, the radiator was fastened directly to the thruster body so that the thermal contact between these two parts increased tenfold. Moreover, the effective surface of the radiator was doubled, resulting in much better heat removal than in the previous case when dedicated heat-guides were applied.

The increase of the magnetic circuit temperature close the Curie temperature influences the magnetic field strength and its topology. To overcome the problem, the material of the magnetic circuit had to be changed. Because of its wider temperature range of operation, the FeCo alloy instead of previously used Armco was applied in the new KLIMT prototype.

\section{Sputtering}

During the tests of the first prototype, the intense sputtering of the chamber material and elements of a magnetic circuit was observed. The sputtering was

Table 1. The basic parameters of pumping system caused by energetic ions produced by the thruster. The sputtered material was deposited mostly at the the cathode support and other thruster elements. However, one can find such a material also on the inner side of the glass windows in the chamber. A semicircular pattern can give additional information about the propagation of ions (see Fig. 4 and Fig. 3b). Unfortunately, it also has negative effects such as decreasing visibility of the plasma plume.

To protect the magnetic poles against sputtering, the design of the main insulator has been changed. The new insulator has been tipped with a cap and a collar to cover the main parts of magnetic circuit.

The previous supporting cathode module was too large and too massive and was strongly sputtered from ions. The new support of the hollow cathode not only reduced the sputtering, but also improved the thruster operation.

\section{Vacuum test facility}

Although the vacuum facility at the IPPLM Electric Propulsion Laboratory (under the Polish acronym PlaNS) for simulating the space environment was designed for the purpose of L $\mu$ PPT project [2], it generates vacuum high enough to provide the conditions for operation of up to more than our class HET. The first ignition was preceded by very accurate studies of vacuum stand efficiency for the controlled krypton mass flow rate and it was confirmed that the volume of vacuum chamber and the efficiency of pumping system are sufficient to be able to carry out the planned experiments.

\section{Vacuum chamber and thruster mounting}

The vacuum chamber for testing plasma thrusters in PlaNS laboratory has the length of $2.3 \mathrm{~m}$ and the inner diameter of $1.2 \mathrm{~m}$. Before starting the experiments, the efficiency of pumping system was measured as a function of the inlet gas mass flow rate. The pumping speed measured for krypton was around $18 \mathrm{~m}^{3} / \mathrm{s}$ for all mass flow rates in the examined range. The parameters of pumping system are presented in Table 1 . The changes of pressure in the chamber vs. mass flow rates of krypton are shown in Fig. 1.

During the efficiency studies, all three pumps were operating. Pressure was measured by Oerlikon-Leybold ITR 90 Ionivac gauge corrected for krypton $(\mathrm{K}=0.5)$. The thruster was fastened to the rear flange by the three added rods (Fig. 2). This flange was mounted on the movable stand allowing for safe and convenient removal of the thruster from the vacuum chamber.

$$
\text { Type of pump }
$$

Pumping speed (for air)

Forevacuum pump (Adixen ACG 600)

Turbomolecular pump (Pfeiffer HiPace 3400 MC)

Cryogenic pump (HSR Velco 900Xe) 


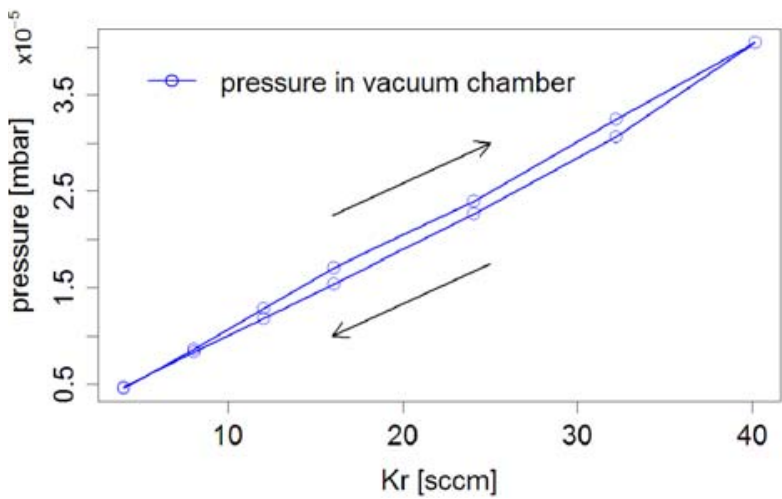

Fig. 1. Ultimate pressure corrected for krypton in vacuum chamber vs. mass flow rate of krypton in sccm. Arrows indicate the flow direction.

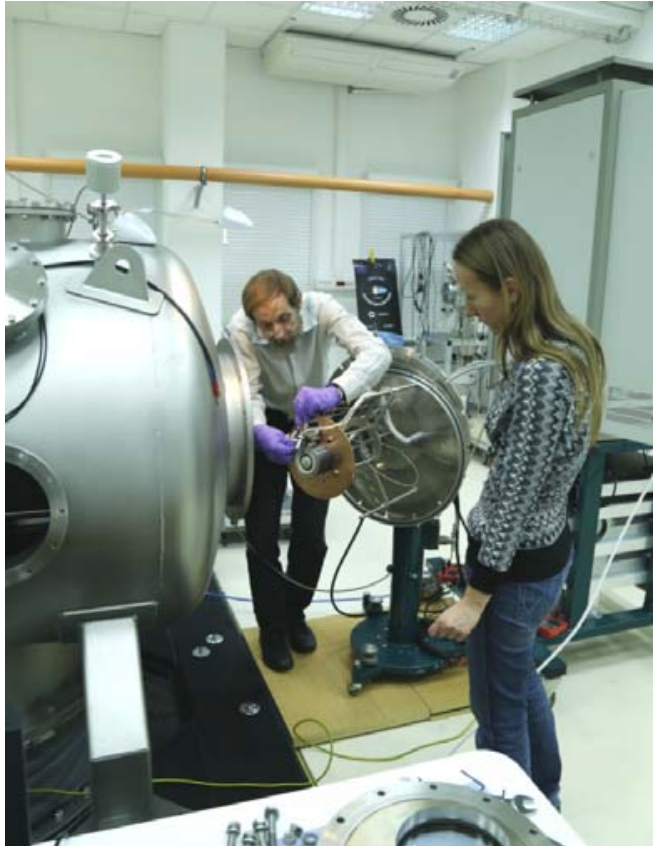

Fig. 2. Vacuum stand for testing plasma thrusters and exposed rear flange.

\section{Gas feeding lines, power units and feedtroughs}

For the purpose of experiments, the gas supply system and a power supply system has been conveniently designed and manufactured. The gas and a part of the electric feed troughs were bolted to the same rear flange as the thruster (Fig. 2). On the air side of the chamber, there are two controllers of gas feeding lines that enable the gas to be supplied to the anode and the cathode separately. At the vacuum side of the chamber, the gas is supplied by $1 / 8$ inch pipelines connected to the feedthrough with Swagelok connectors.

During the first tests, it turned out that the anode cable must be connected through a different flange via special high-voltage feedthrough. A four-pin feedthrough rated up to $20 \mathrm{kV} / 25 \mathrm{~A}$ was used for anode and keeper connections. The other cables (for heater and coils) were connected by low-voltage Lemo-type plugs. At the vacuum side, all the cables were protected with fiberglass tubings and in the hottest regions with alumina beads.
Table 2. Krypton mass flow rates in [mg/s] and in [ $\mathrm{sccm}]$ (standard cubic centimeters per second), sccm as well as the corresponding (by number density) xenon mass flow rates

\begin{tabular}{cc}
\hline Krypton mass flow rate & $\begin{array}{c}\text { Corresponding xenon } \\
\text { mass flow rate }\end{array}$ \\
\hline $0.75 \mathrm{mg} / \mathrm{s} \approx 12.93 \mathrm{sccm}$ & $1.18 \mathrm{mg} / \mathrm{s}$ \\
$1.00 \mathrm{mg} / \mathrm{s} \approx 17.24 \mathrm{sccm}$ & $1.57 \mathrm{mg} / \mathrm{s}$ \\
$1.25 \mathrm{mg} / \mathrm{s} \approx 21.55 \mathrm{sccm}$ & $2 \mathrm{mg} / \mathrm{s}$ \\
\hline
\end{tabular}

\section{Measurements campaign}

The final location of the hollow cathode was established, with the help of finite element method magnetics (FEMM) [3] program, which modeled electrostatic field configuration, as expected, just before the ignition. Thus, the distance of the cathode orifice from the thruster axis was set at $36 \mathrm{~mm}$, while its distance from the thruster exit plane at $10 \mathrm{~mm}$. The anode was moved toward the exit plane, shortening the channel length to $18 \mathrm{~mm}$. This new configuration led to much more stable thruster operation. However, the position of the cathode was not optimized from the point of view of the thruster efficiency, mainly because of lack of time. Therefore, the same configuration was used in all of the subsequent measurement series.

Mass flow rates of krypton that were used in the measurement campaign are listed in Table 2. The first one equal to $0.75 \mathrm{mg} / \mathrm{s}$ was chosen because it corresponds (by number density) to $1.18 \mathrm{mg} / \mathrm{s}$ of xenon. This xenon mass flow rate provided the most stable thruster operation in the previous campaign that was performed it ESA Propulsion Laboratory (EPL) with the use of the first prototype. Similarly, the second mass flow rate $(1.00 \mathrm{mg} / \mathrm{s})$ was chosen, because it corresponds to xenon mass flow rate of $1.57 \mathrm{mg} / \mathrm{s}$. This value was also used during the campaign at EPL and it resulted in the maximal thrust equal to $20 \mathrm{mN}$ [4]. The last value of mass flow rate, $1.25 \mathrm{mg} / \mathrm{s}$, was chosen to study the behavior of the thruster under extreme heat loads.

\section{Current and voltage measurements}

At first, it was necessary to ascertain the best conditions under which the thruster operates reliably and the discharge is stable. To determine the operating envelope of the thruster, the studies of its current-voltage characteristics for several values of the mass flow rate were performed. Also a series of tests with various magnetic field configurations were made. The following optimal parameters for cathode operation were established: 3-3.5 A for the keeper current and $3 \mathrm{sccm}$ of krypton mass flow rate. These parameters provide sufficient electron current and stable operation of the thruster. It is worth noting that in these conditions the heater current could be set to zero and the thruster could be ignited when the nominal magnetic field was already switched on (hard ignition). With the aforementioned pa- 
rameters, the thruster is reliably operating in the constant-voltage regime.

A Tektronix DPO4104B digital oscilloscope was used for recording the discharge current waveforms. Coil currents and corresponding voltages were systematically recorded. This information was used for the calculation of coil resistance from coil temperatures, which approximate the inner temperatures of the thruster body [5].

\section{The thrust measurement}

The graphite target with a diameter of $20 \mathrm{~cm}$ and width of $2 \mathrm{~cm}$ was installed at the thrust balance, manufactured by MECARTEX company. The thrust balance was located $90 \mathrm{~cm}$ from the thruster exit plane (the assembly is shown in Fig. 3a). The role of graphite target was to capture the part of momentum of the stream of particles generated by the thruster. It allowed us to estimate the thrust produced by our HET.

A thrust balance is a kind of harmonic oscillator in which deflection with respect to the equilibrium position is proportional to the applied force - in our case, the force $T$ exerted on the graphite target. Nevertheless, we observe long-term drift of the

a

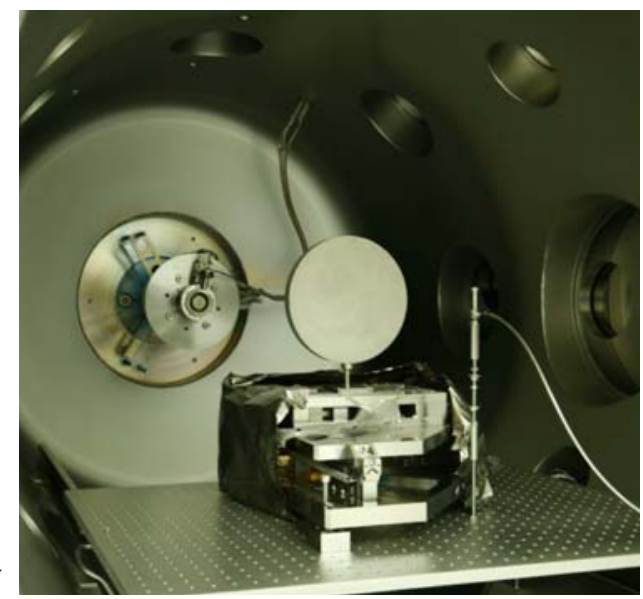

b

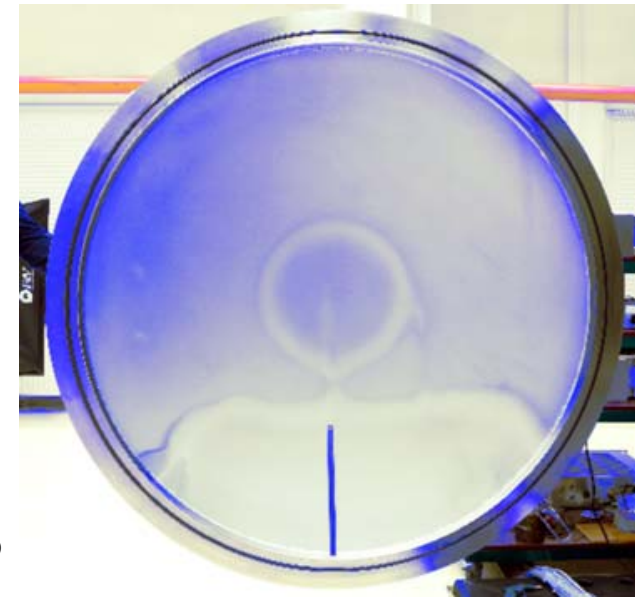

Fig. 3. (a) The target on the thrust balance in a vacuum chamber. (b) Two circles on the center of sputtered chamber doors. The smaller circle has a diameter of around $30 \mathrm{~cm}$. The color balance was changed to emphasize the effect of sputtering traces. equilibrium position in time even if the thruster is not operating. Therefore, we set the thrust measurement time much shorter than characteristic time of the drift. Typically our measurement time of the thrust was chosen to be $1.5 \mathrm{~min}$. This relatively short measurement time allows us to estimate that the error caused by long-term drift does not exceed $5 \%$.

The measurements were conducted for different values of the thruster operating parameters (discharge voltages, mass flow rates and the ratio of currents in the inner and outer coil of the thruster magnetic circuit). Each measurement series was completed when the temperature of the inner coil reached $500^{\circ} \mathrm{C}$.

\section{Experimental results}

In the search for the optimal operating parameters, several inner to outer coil current ratios were thoroughly examined. Despite the fact that the magnetic circuit was designed to be able to produce radial component of induction exceeding $200 \mathrm{Gs}$, it appeared that such a strong magnetic field is not necessary and, if applied, may result in the unstable thruster operation. The experiments have shown that one of the best coil current ratio in terms of maximum thrust production is $0.6 / 0.5$ for higher tested discharge voltages. That is why most of the measurements were performed with this setting.

The operating envelope of the thruster has been investigated in a wide range of voltages. It was established that thruster operates most stably in the $180-260 \mathrm{~V}$ range. Also, in this range, the modulation of the discharge current usually does not exceed $25 \%$ and can be even less than $10 \%$ for the mass flow rate not less than $1 \mathrm{mg} / \mathrm{s}$ of krypton. It seems that the preferred voltage is close to $240 \mathrm{~V}$, which can be called the nominal voltage. Most experiments were performed for this value.

\section{Thrust estimation}

The force $T$ on the target that was recorded for different values of discharge voltage $U_{d}$ and constant coil current ratio is depicted in Fig. 5. It is a collection of data from several measurement series. The different colors of experimental points indicate different temperatures of the inner coil.

To calculate the thrust basing on the force exerted on the graphite target, one needs to know the angular distribution of the plasma stream. In our experiment, this distribution was not measured. However, knowing the typical angular distributions from the literature and the position and size of our graphite target, we can estimate that it captures about 30\% of the plasma beam.

The maximal value of normalized thrust $T$, or rather force that is exerted on the graphite target, is close to $6 \mathrm{mN}$. Assuming that it is 30\% of the thrust produced by HET, we can fix it at $\sim 20 \mathrm{mN}$. Thus we expect that the corresponding specific impulse should be close to $1500 \mathrm{~s}$. 

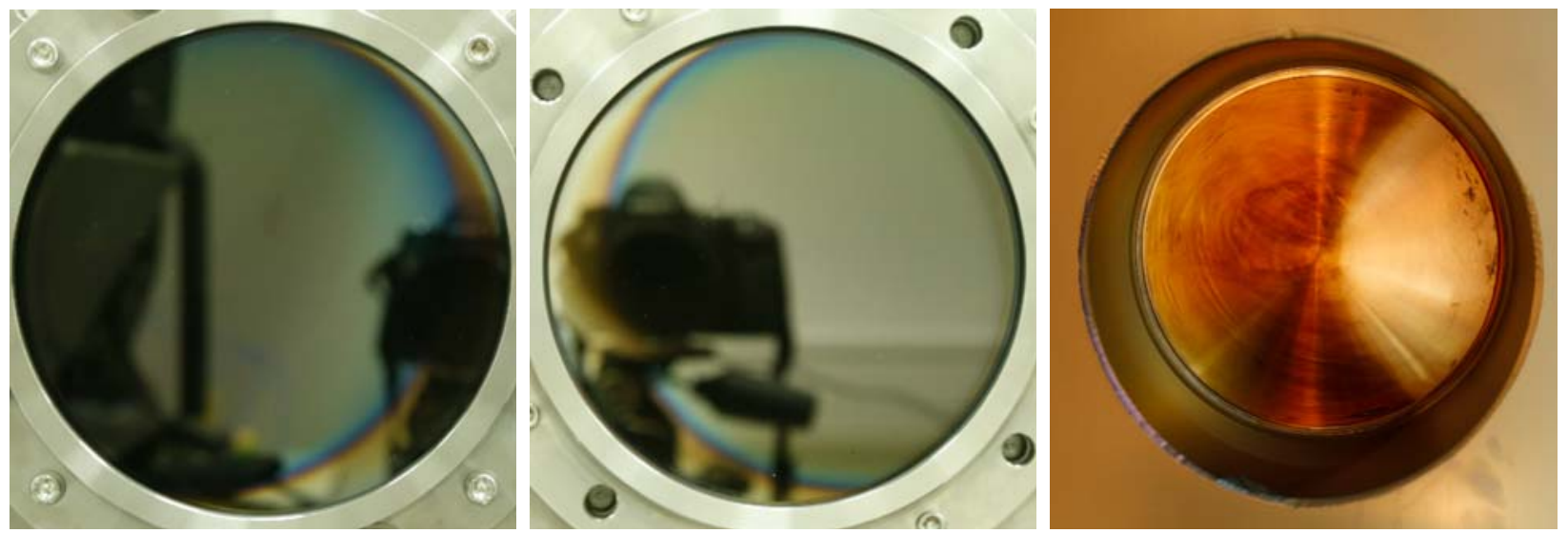

Fig. 4. Effects caused by the plasma stream. Two pictures of the shady window and a picture of the inner flange. On this basis, it is possible to study an angular distribution of the deposited particles.
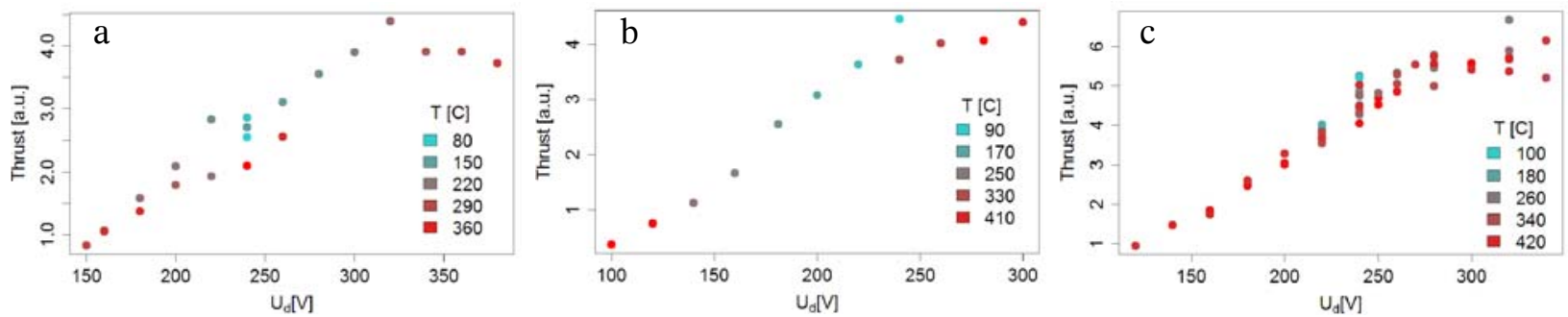

Fig. 5. Normalized thrust as measured for coil current ratio $0.6 / 0.5$ and different voltages for mass flow rate equal to: (a) $0.75 \mathrm{mg} / \mathrm{s}$, (b) $1.00 \mathrm{mg} / \mathrm{s}$, and (c) $1.25 \mathrm{mg} / \mathrm{s}$.
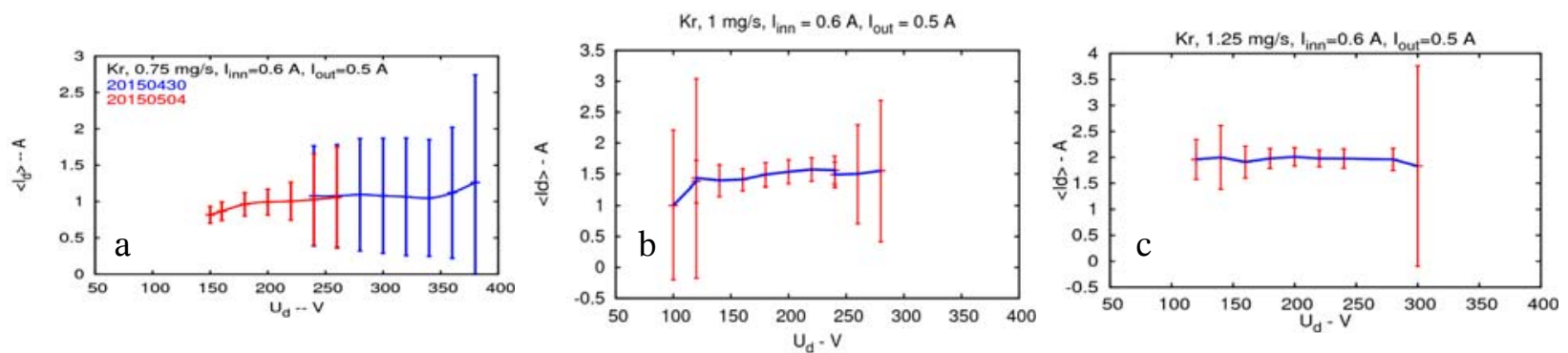

Fig. 6. Current-voltage characteristics for Hall thruster when operated at coil current $I_{\text {in }}=0.6 \mathrm{~A}, I_{\text {out }}=0.5 \mathrm{~A}$ and krypton mass flow rate equal to (a) $0.75 \mathrm{mg} / \mathrm{s}$, (b) $1.00 \mathrm{mg} / \mathrm{s}$, (c) $1.25 \mathrm{mg} / \mathrm{s}$. These plots were prepared by J. Kurzyna [6].

\section{Current-voltage characteristics}

In Fig. 6, the current-voltage characteristics for mean value of discharge current are reproduced. The bars correspond to the standard deviation of the current oscillations. In the range of small voltages, current oscillations are irregular and low, whereas in the range of larger voltages, oscillations are more

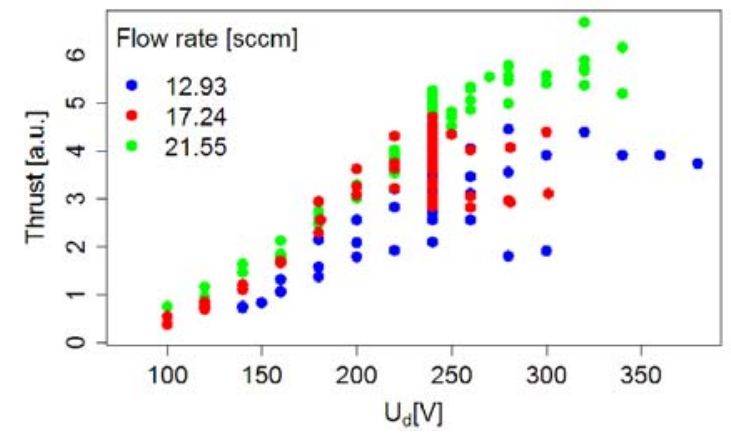

Fig. 7. Cumulative plot of the normalized thrust vs. discharge current as measured in all test series. regular with an apparent deeply modulated current mode. In the large-voltage regime, the frequency of the breathing mode increases, displaying a typical behavior of the curve for this kind of Hall thrusters. Current-voltage plots for krypton correspond to the known I-V characteristics reproduced for xenon [4]. The inclinations of I-V characteristics are very small in the region of weak discharge current modulation, resulting in almost constant mean value of the discharge current.

\section{Conclusions}

The measurement campaign aiming for the evaluation of the second KLIMT prototype version has been performed at IPPLM's new EP laboratory, which appeared to be equipped well enough for testing $\sim 500 \mathrm{~W}$ class HETs. The thruster performance as well as its design (taking into account such factors as the resistance to overheating of the thruster elements and magnetic circuit material) were subjected 
to assessment. Effective troubleshooting of the overheating in gas feeding lines and electric flush-overs at the surface of HV feedthrough insulators as well as adjustment of the KLIMT's discharge channel geometry (by positioning the cathode and the anode with respect to the thruster exit plane) resulted in stable KLIMT's functioning in the wide range of operating parameters. The results obtained during these studies confirm the original design assumptions, e.g., magnetic field topology as well as the improvements introduced in the second prototype of the thruster. For instance, the new solution for the main insulator effectively protects the poles of magnetic circuit against sputtering.

Having assured reliable thruster functioning, the operating envelope was examined to indicate the nominal parameters in terms of discharge voltage, released power, magnetic field topology, and thrust production. Due to the lack of absolute thrust measurement (and thus the specific impulse), we were not able to calculate the thruster efficiency in terms of output to input power ratio. However, our indirect method for thrust magnitude assessment was sufficient to reveal the range of good thruster performance, including the influence of the increase in time and temperature. Nevertheless, the precise thrust measurements for krypton propellant are still lacking and they are scheduled to be performed during the experiments in EPL at ESTEC within a few months.
This work was performed at the Institute of Plasma Physics and Laser Microfusion in Warsaw, Poland.

\section{References}

1. Biagioni, L., Saverdi, M., Berti, M., Cesari, U., \& Andrenucci, M. (2003). Design and preliminary characterization of a $5 \mathrm{~kW}$ Hall thruster prototype. (Report IEPC-03-228).

2. Barral, S., Kurzyna, J., Remírez, E., Martín, R., Ortiz, P., Alonso, J., Bottinelli, S., Mabillard, Y., Zaldíva, A., Rangsten, P., \& Koppel, C. R. (2013). Development status of an open capilla pulsed plasma thruster with non-volatile liquid propellant. In Proceedings of the 33rd International Electric Propulsion Conference, The George Washington University, Washington, D.C., USA, October 6-10, 2013. (Report IEPC-2013-291).

3. Finite element method magnetics. Retrieved from www.femm.info/wiki/HomePage.

4. Kurzyna, J., Barral, S., Daniłko, D., Miedzik, J., Bulit, A., \& Dannenmayer, K. (2013). First tests of the KLIMT thruster with xenon propellant at the ESA Propulsion Laboratory. Warsaw: IPPLM. (Internal report).

5. Kurzyna, J., Barral, S., Daniłko, D., \& Miedzik, J. (2015). KLIMPT - Krypton Large Impulse Thruster, Deliverable 3.2, First Prototype Design and Testing Report. Warsaw: IPPLM. (Internal report).

6. Kurzyna, J., Daniłko, D., Szelecka, A., \& Bourdain, L. (2015). KLIMPT - Krypton Large Impulse Thruster, Deliverable 3.4, Second Prototype Design and Testing Report. Warsaw: IPPLM. (Internal report). 